\title{
Energy decay and nonexistence of solution for a reaction-diffusion equation with exponential nonlinearity
}

\author{
Huiya Dai ${ }^{1,2^{*}}$ and Hongwei Zhang ${ }^{2}$
}

\section{"Correspondence:}

54214383@qq.com

${ }^{1}$ School of Mathematics and

Statistics, Xi'an Jiaotong University,

Xianning Road, Xi'an, P.R. China

${ }^{2}$ Department of Mathematics,

Henan University of Technology,

Lianhua Street, Zhengzhou,

P.R. China

\begin{abstract}
In this work we consider the energy decay result and nonexistence of global solution for a reaction-diffusion equation with generalized Lewis function and nonlinear exponential growth. There are very few works on the reaction-diffusion equation with exponential growth $f$ as a reaction term by potential well theory. The ingredients used are essentially the Trudinger-Moser inequality.
\end{abstract}

Keywords: reaction-diffusion equation; stable and unstable set; exponential reaction term; decay rate; global nonexistence

\section{Introduction}

In this paper, we study the following initial boundary value problem with generalized Lewis function $a(x, t)$ which depends on both spacial variable and time:

$$
\begin{aligned}
& a(x, t) u_{t}-\Delta u=f(u), \quad x \in \Omega, t>0, \\
& u(x, t)=0, \quad x \in \partial \Omega, t>0, \\
& u(x, 0)=u_{0}(x), \quad x \in \Omega,
\end{aligned}
$$

here $f(s)$ is a reaction term with exponential growth at infinity to be specified later, $\Omega$ is a bounded domain with smooth boundary $\partial \Omega$ in $R^{2}$.

For the reaction-diffusion equation with polynomial growth reaction terms (that is, equation (1) with $a(x, t)=1$ and $\left.f(u)=|u|^{p-1} u\right)$, there have been many works in the literature; one can find a review of previous results in $[1,2]$ and references therein, which are not listed in this paper just for concision. Problem (1)-(3) with $a(x, t)>0$ describes the chemical reaction processes accompanied by diffusion [2]. The author of work [1] proved the existence and asymptotic estimates of global solutions and finite time blow-up of problem (1)-(3) with $a(x, t)>0$ and the critical Sobolev exponent $p=\frac{n+2}{n-2}$ for $f(u)=u^{p}$.

In this paper we assume that $f(s)$ is a reaction term with exponential growth like $e^{s^{2}}$ at infinity. When $a(x, t)=1, f(u)=e^{u}$, model (1)-(3) was proposed by [3] and [4]. In this case, Fujita [5] studied the asymptotic stability of the solution. Peral and Vazquez [6] and Pulkkinen [7] considered the stability and blow-up of the solution. Tello [8] and Ioku [9] considered the Cauchy problem of heat equation with $f(u) \approx e^{u^{2}}$ for $|u| \geq 1$.

Recently, Alves and Cavalcanti [10] were concerned with the nonlinear damped wave equation with exponential source. They proved global existence as well as blow-up of so-

\section{Springer}

@2014 Dai and Zhang; licensee Springer. This is an Open Access article distributed under the terms of the Creative Commons Attribution License (http://creativecommons.org/licenses/by/2.0), which permits unrestricted use, distribution, and reproduction in any medium, provided the original work is properly cited. 
lutions in finite time by taking the initial data inside the potential well [11]. Moreover, they also got the optimal and uniform decay rates of the energy for global solutions.

Motivated by the ideas of $[1,10]$, we concentrate on studying the uniform decay estimate of the energy and finite time blow-up property of problem (1)-(3) with generalized Lewis function $a(x, t)$ and exponential growth $f$ as a reaction term. To the authors' best knowledge, there are very few works in the literature that take into account the reactiondiffusion equation with exponential growth $f$ as a reaction term by potential well theory. The majority of works in the literature make use of the potential well theory when $f$ possesses polynomial growth. See, for instance, the works [12-16] and a long list of references therein. The ingredients used in our proof are essentially the Trudinger-Moser inequality $($ see $[17,18])$. We establish decay rates of the energy by considering ideas from the work of Messaoudi [15]. The case of nonexistence results is also treated, where a finite time blowup phenomenon is exhibited for finite energy solutions by the standard concavity method adapted for our context.

The remainder of our paper is organized as follows. In Section 2 we present the main assumptions and results, Section 3 and Section 4 are devoted to the proof of the main results.

Throughout this study, we denote by $\|\cdot\|,\|\cdot\|_{p},\|\cdot\|_{H_{0}^{1}}$ the usual norms in spaces $L^{2}(\Omega)$, $L^{p}(\Omega)$ and $H_{0}^{1}(\Omega)$, respectively.

\section{Assumptions and preliminaries}

In this section, we present the main assumptions and results. We always assume that:

(A1) $a(x, t)$ is a positive differentiable function and is bounded for $t \in[0,+\infty), x \in \Omega$.

(A2) $f: R \rightarrow R$ is a $C^{1}$ function. The function $f(t) / t$ is increasing in $(0, \infty)$, and for each $\beta>0$, there exists a positive constant $C_{\beta}$ such that

$$
|f(t)| \leq C_{\beta} e^{\beta t^{2}}, \quad\left|f^{\prime}(t)\right| \leq C_{\beta} e^{\beta t^{2}}
$$

(A3) For each $\varepsilon>0, \beta>0$ and $p>1$ fixed, there exists a positive constant $C(\varepsilon, \beta)$ such that

$$
\begin{aligned}
& |f(t)| \leq \varepsilon|t|+C(\varepsilon, \beta)|t|^{p-1} e^{\beta t^{2}}, \\
& |F(t)| \leq \varepsilon|t|^{2}+C(\varepsilon, \beta)|t|^{p} e^{\beta t^{2}}
\end{aligned}
$$

where $F(t)=\int_{0}^{t} f(s) d s$.

(A4) There exists a positive constant $\theta>2$ such that

$$
0<\theta F(t)<f(t) t, \quad t \in R /\{0\} .
$$

A typical example of functions satisfies (A2)-(A4) is $f(t)=C|t|^{p-1} t e^{M t^{\alpha}}$, with given $p>1$, $M>0, C>0$, and $\alpha \in(1,2)$.

Now we define some functional as follows:

$$
\begin{aligned}
& E(t)=E(u)=\frac{1}{2}\|\nabla u\|^{2}-\int_{\Omega} F(u) d x, \\
& I(t)=I(u)=\|\nabla u\|^{2}-\int_{\Omega} u f(u) d x,
\end{aligned}
$$


then the 'potential depth' given by

$$
d=\inf \left\{\sup _{\lambda \in R} E(\lambda u), u \in H_{0}^{1} /\{0\}\right\}
$$

is a positive constant [10]. Hence, we are able to define stable and unstable sets respectively as follows:

$$
\begin{aligned}
& W_{1}=\left\{u \in H_{0}^{1}, E(u)<d, I(u)>0\right\}, \\
& W_{2}=\left\{u \in H_{0}^{1}, E(u)<d, I(u)<0\right\} .
\end{aligned}
$$

We also need the following lemmas.

Lemma 2.1 $[17,18]$ Let $\Omega$ be a bounded domain in $R^{2}$. For all $u \in H_{0}^{1}(\Omega)$,

$$
e^{\alpha|u|^{2}} \in L^{1}(\Omega) \text { for all } \alpha>0,
$$

and there exist positive constants $m_{2}$ such that

$$
\sup _{u \in H_{0}^{1}(\Omega),\|\nabla u\| \leq 1} \int_{\Omega} e^{\alpha|u|^{2}} d x=m_{2}<\infty \quad \text { for all } \alpha \leq 4 \pi .
$$

Lemma 2.2 [19] Let $\phi(t)$ be a nonincreasing and nonnegative function on $[0, \infty)$, such that

$$
\sup _{s \in[t, t+1]} \phi(s) \leq C(\phi(t)-\phi(t+1)), \quad t>0,
$$

then

$$
\phi(t) \leq C e^{-\omega t}
$$

where $C, \omega$ are positive constants depending on $\phi(0)$ and other known qualities.

Lemma 2.3 [20] Suppose that a positive, twice-differentiable function $H(t)$ satisfies on $t \geq 0$ the inequality

$$
H^{\prime \prime}(t) H(t)-(\delta+1)\left(H^{\prime}(t)\right)^{2} \geq 0,
$$

where $\delta>0$, then there is $t_{1}<t_{2}=\frac{H(0)}{\delta H^{\prime}(0)}$ such that $H(t) \rightarrow \infty$ as $t \rightarrow t_{1}$.

In order to state and prove our main results, we remind that by the embedding theorem there exists a constant $C_{0}$ depending on $p$ and $\Omega$ only such that

$$
\|u\|_{p} \leq C_{0}\|\nabla u\|
$$

By multiplying equation (1) by $u_{t}$, integrating over $\Omega$, using integration by parts and $a(x, t)>0$, we get

$$
E^{\prime}(t)=-\int_{\Omega} a(x, t) u_{t}^{2}(x, t) d x \leq 0 .
$$

Our main results read as follows. 
Theorem 2.1 Let (A1)-(A4) hold. Assume further that $u_{0} \in W_{1}$ satisfies

$$
\varepsilon_{0} C_{0}^{2}+C_{\varepsilon_{0}} C_{0}^{p} m_{2}^{\frac{1}{2}}\left(\frac{2 \theta}{\theta-2} E(0)\right)^{p-2}<1
$$

for some sufficiently small $\varepsilon_{0}>0$ and $C_{\varepsilon_{0}}>0$. Then there exist positive constants $K$ and $k$ such that the energy $E(t)$ satisfies the decay estimates for large $t$

$$
E(t) \leq K e^{-k t}
$$

Theorem 2.2 Let (A1)-(A4) hold. Assume further that $a_{t}(x, t) \leq 0, u_{0} \in W_{2}$ and $E(0)<$ $\frac{(\theta-2) d}{\theta}<d$, then the solutions of (1)-(3) blow up in finite time.

\section{Proof of decay of the energy}

In this section we prove Theorem 2.1. We divide the proof into two lemmas.

Lemma 3.1 Under the assumptions of Theorem 2.1, we have, for all $t \geq 0, u(t) \in W_{1}$.

Proof Since $I\left(u_{0}\right) \geq 0$, then there exists (by continuity) $T_{m}<T$ such that

$$
I(u(t)) \geq 0, \quad \forall t \in\left[0, T_{m}\right] .
$$

This and (A4) give

$$
\begin{aligned}
E(t) & =\left(\frac{1}{2}-\frac{1}{\theta}\right)\|\nabla u\|^{2}+\frac{1}{\theta} I(u)+\int_{\Omega}\left(\frac{1}{\theta} u f(u)-F(u)\right) d x \\
& \geq\left(\frac{1}{2}-\frac{1}{\theta}\right)\|\nabla u\|^{2}, \quad \forall t \in\left[0, T_{m}\right] .
\end{aligned}
$$

So, by (15) we have

$$
\|\nabla u\|^{2} \leq \frac{2 \theta}{\theta-2} E(t) \leq \frac{2 \theta}{\theta-2} E(0) \leq \frac{2 \theta d}{\theta-2}, \quad \forall t \in\left[0, T_{m}\right] .
$$

We then use (5), the Holder inequality and the embedding theorem to obtain, for each $t \in\left[0, T_{m}\right]$,

$$
\begin{aligned}
\int_{\Omega} u f(u) d x & \leq \int_{\Omega}\left[\varepsilon|u|^{2}+C(\varepsilon, \beta)|u|^{p} e^{\beta u^{2}}\right] d x \\
& \leq \varepsilon C_{0}^{2}\|\nabla u\|^{2}+C(\varepsilon, \beta)\left(\int_{\Omega}|u|^{2 p} d x\right)^{\frac{1}{2}}\left(\int_{\Omega} e^{2 \beta u^{2}} d x\right)^{\frac{1}{2}} .
\end{aligned}
$$

Once $\|\nabla u\|^{2} \leq \frac{2 \theta d}{\theta-2}$, we choose $\beta$ such that $\frac{\theta \beta d}{\theta-2}<\pi$, then, from Trudinger-Moser inequality (11),

$$
\int_{\Omega} e^{2 \beta|u|^{2}} d x \leq \int_{\Omega} e^{2 \beta\|\nabla u\|^{2}\left(\frac{u}{\|\nabla u\|}\right)^{2}} d x \leq m_{2},
$$


and therefore, by (16) for $\varepsilon_{0}>0$ and $C_{\varepsilon_{0}}>0$, we have

$$
\begin{aligned}
\int_{\Omega} u f(u) d x & \leq \varepsilon_{0} C_{0}^{2}\|\nabla u\|^{2}+C_{\varepsilon_{0}} C_{0}^{p} m_{2}^{\frac{1}{2}}\|\nabla u\|^{p} \\
& =\varepsilon_{0} C_{0}^{2}\|\nabla u\|^{2}+C_{\varepsilon_{0}} C_{0}^{p} m_{2}^{\frac{1}{2}}\left(\frac{2 \theta}{\theta-2} E(t)\right)^{p-2}\|\nabla u\|^{2} \\
& \leq\left[\varepsilon_{0} C_{0}^{2}+C_{\varepsilon_{0}} C_{0}^{p} m_{2}^{\frac{1}{2}}\left(\frac{2 \theta}{\theta-2} E(0)\right)^{p-2}\right]\|\nabla u\|^{2}<\|\nabla u\|^{2} .
\end{aligned}
$$

By virtue of (21) and the definition of $I(t)$, we have

$$
I(t)=\|\nabla u\|^{2}-\int_{\Omega} u f(u) d x>0 .
$$

This shows that $u(t) \in W_{1}$ for all $t \in\left[0, T_{m}\right]$. By repeating this procedure and the fact that $E(t) \leq E(0)$, we obtain

$$
\lim _{t \rightarrow T_{m}}\left(\varepsilon_{0} C_{0}^{2}+C_{\varepsilon_{0}} C_{0}^{p} m_{2}^{\frac{1}{2}}\left(\frac{2 \theta}{\theta-2} E(t)\right)^{p-2}\right) \leq \rho<1 .
$$

This is extended to $T$.

Lemma 3.2 Under the assumptions of Theorem 2.1, we have, for $\eta=1-\left[\varepsilon_{0} C_{0}^{2}+C_{\varepsilon_{0}} C_{0}^{p} m_{2}^{\frac{1}{2}} \times\right.$ $\left.\left(\frac{2 \theta}{\theta-2} E(0)\right)^{p-2}\right]$,

$$
\eta\|\nabla u\|^{2}<I(t)
$$

Proof It suffices to rewrite (21) as

$$
\begin{aligned}
\int_{\Omega} u f(u) d x & \leq\left[\varepsilon_{0} C_{0}^{2}+C_{\varepsilon_{0}} C_{0}^{p} m_{2}^{\frac{1}{2}}\left(\frac{2 \theta}{\theta-2} E(0)\right)^{p-2}\right]\|\nabla u\|^{2} \\
& =(1-\eta)\|\nabla u\|^{2}=\|\nabla u\|^{2}-\eta\|\nabla u\|^{2} .
\end{aligned}
$$

Thus (22) follows from (23).

Proof of Theorem 2.1 We integrate (15) over $[t, t+1]$ to obtain

$$
E(t)-E(t+1)=\int_{t}^{t+1} \int_{\Omega} a(x, s) u_{t}^{2}(x, s) d x d s=D^{2}(t) .
$$

Now we multiply (1) by $u$ and integrate over $\Omega \times[t, t+1]$ to arrive at

$$
\begin{aligned}
\int_{t}^{t+1} I(s) d s & =\int_{t}^{t+1}\left[\|\nabla u\|^{2}-\int_{\Omega} u f(u) d x\right] d s \\
& =\int_{t}^{t+1} \int_{\Omega} a(x, t) u_{t}(x, t) u(x, t) d x d s \\
& \leq A \int_{t}^{t+1}\left\|a^{\frac{1}{2}} u_{t}(s)\right\|\|u(s)\| d s,
\end{aligned}
$$


where $A^{2}=\sup _{(x, t) \in \Omega \times[0,+\infty)}|a(x, t)|$. Exploiting (14) and (19), we obtain

$$
\begin{aligned}
\int_{t}^{t+1} I(s) d s & \leq A C_{0}\left(\frac{2 \theta}{\theta-2}\right)^{\frac{1}{2}}\left(\sup _{s \in[t, t+1]} E^{\frac{1}{2}}(t)\right) \int_{t}^{t+1}\left\|a^{\frac{1}{2}} u_{t}\right\| d s \\
& \leq A C_{0}\left(\frac{2 \theta}{\theta-2}\right)^{\frac{1}{2}}\left(\sup _{s \in[t, t+1]} E^{\frac{1}{2}}(t)\right) D(t) .
\end{aligned}
$$

Using (7), (23) and (22), we have

$$
\begin{aligned}
E(t) & =\frac{\theta-2}{2 \theta}\|\nabla u\|^{2}+\frac{1}{\theta} I(t)+\int_{\Omega}\left(\frac{1}{\theta} u f(u)-F(u)\right) d x \\
& \leq \frac{\theta-2}{2 \theta}\|\nabla u\|^{2}+\frac{1}{\theta} I(t)+\int_{\Omega} \frac{2}{\theta} u f(u) d x \\
& \leq \frac{\theta-2}{2 \theta}\|\nabla u\|^{2}+\frac{1}{\theta} I(t)+\frac{2}{\theta}(1-\eta)\|\nabla u\|^{2} \\
& \leq\left[\frac{\theta-2}{2 \theta \eta}+\frac{1}{\theta}+\frac{2}{\theta}(1-\eta)\right] I(t) .
\end{aligned}
$$

Integrating both sides of (27) over $[t, t+1]$ and using (26), one can write

$$
\int_{t}^{t+1} E(s) d s=\left[\frac{\theta-2}{2 \theta \eta}+\frac{1}{\theta}+\frac{2}{\theta}(1-\eta)\right] A C_{0}\left(\frac{2 \theta}{\theta-2}\right)^{\frac{1}{2}}\left(\sup _{s \in[t, t+1]} E^{\frac{1}{2}}(t)\right) D(t) .
$$

By using (15) again, we have $E(s) \geq E(t+1), \forall s \leq t+1$, hence

$$
\int_{t}^{t+1} E(s) d s \geq E(t+1)
$$

Inserting (29) in (24) and using (27), we easily have

$$
\begin{aligned}
E(t) & \leq \int_{t}^{t+1} E(s) d s+\int_{t}^{t+1} \int_{\Omega} a(x, s) u_{t}^{2}(x, s) d x d s \\
& \leq\left[\frac{\theta-2}{2 \theta \eta}+\frac{1}{\theta}+\frac{2}{\theta}(1-\eta)\right] A C_{0}\left(\frac{2 \theta}{\theta-2}\right)^{\frac{1}{2}}\left(\sup _{s \in[t, t+1]} E^{\frac{1}{2}}(t)\right) D(t)+D^{2}(t) \\
& \leq C_{1}\left[E^{\frac{1}{2}}(t) D(t)+D^{2}(t)\right]
\end{aligned}
$$

for $C_{1}$ a constant depending on $C_{0}, A, \theta, \eta$ only. We then use Young's inequality to get from (30) and (24)

$$
\sup _{s \in[t, t+1]} E(t) \leq C_{2} D^{2}(t) \leq C_{2}(E(t)-E(t+1))
$$

By (12) in Lemma 2.2 we then get the results.

\section{Proof of the blow-up result}

In this section, we shall prove Theorem 2.2 by adapting the concavity method (see Levine [20]). We recall the following lemma in [10]. 
Lemma 4.1 [10] Assume that $u_{0} \in W_{2}$ and $E(0)<d$, then it holds that

$$
\begin{aligned}
& u(t) \in W_{2} \quad \text { for } t \in\left[0, T_{\max }\right), \\
& \|\nabla u\|^{2} \geq 2 d \quad \text { for } t \in\left[0, T_{\max }\right) .
\end{aligned}
$$

Proof of Theorem 2.2 Assume by contradiction that the solution is global. Then, for any $T>0$, we consider the function $H(\cdot):[0, T] \rightarrow R^{+}$defined by

$$
\begin{aligned}
H(t)= & \int_{0}^{t} \int_{\Omega} a(x, s) u^{2}(x, s) d x d s+\int_{0}^{t} \int_{\Omega}(s-t) a_{t}(x, s) u^{2}(x, s) d x d s \\
& +(T-t) \int_{\Omega} a(x, 0) u_{0}^{2}(x) d x+\rho\left(t+t_{0}\right)^{2},
\end{aligned}
$$

where $t_{0}, T, \rho$ are positive constants which will be fixed later. Direct computations show that

$$
\begin{aligned}
H^{\prime}(t) & =\int_{\Omega} a(x, t) u^{2}(x, t) d x-\int_{0}^{t} \int_{\Omega} a_{t}(x, s) u^{2}(x, s) d x d s-\int_{\Omega} a(x, 0) u_{0}^{2} d x+2 \rho\left(t+t_{0}\right) \\
& =2 \int_{0}^{t} \int_{\Omega} a(x, s) u_{t}(x, s) u(x, s) d x d s+2 \rho\left(t+t_{0}\right) \\
H^{\prime \prime}(t) & =2 \int_{\Omega} a(x, t) u(x, t) u_{t}(x, s) d x+2 \rho
\end{aligned}
$$

Then, due to equations (1), (7) and (33), we have

$$
\begin{aligned}
H^{\prime \prime}(t) & =-2\|\nabla u\|^{2}+2 \int_{\Omega} u f(u) d x+2 \rho \\
& \geq-2\|\nabla u\|^{2}+2 \theta \int_{\Omega} F(u) d x+2 \rho=(\theta-2)\|\nabla u\|^{2}-2 \theta E(t)+2 \rho \\
& =(\theta-2)\|\nabla u\|^{2}-2 \theta E(0)+2 \theta \int_{0}^{t} \int_{\Omega} a(x, s) u_{t}^{2}(x, s) d x d s+2 \rho \\
& \geq 2(\theta-2) d-2 \theta E(0)+2 \theta \int_{0}^{t} \int_{\Omega} a(x, s) u_{t}^{2}(x, s) d x d s+2 \rho .
\end{aligned}
$$

Now we take $0<\rho<\frac{(\theta-2) d-\theta E(0)}{\theta-1}$ such that $2(\theta-2) d-2 \theta E(0)+2 \rho>2 \theta \rho$ (this $\rho$ can be chosen since $\left.E(0)<\frac{(\theta-2) d}{\theta}\right)$, and then

$$
H^{\prime \prime}(t) \geq 2 \theta \rho+2 \theta \int_{0}^{t} \int_{\Omega} a(x, s) u_{t}^{2}(x, s) d x d s
$$

We also note that

$$
\begin{aligned}
& H(0)=T \int_{\Omega} a(x, 0) u_{0}^{2}(x) d x+\rho t_{0}^{2}>0, \\
& H^{\prime}(0)=2 \rho t_{0}>0, \\
& H^{\prime \prime}(t) \geq 2 \theta \rho>0, \quad t \geq 0 .
\end{aligned}
$$


Therefore $H(t)$ and $H^{\prime}(t)$ are both positive. Since $a_{t}(x, t) \leq 0$ for all $x \in \Omega$ and $t \geq 0$, by the construction of $H(t)$, it is clear that

$$
H(t) \geq \int_{0}^{t} \int_{\Omega} a(x, s) u^{2}(x, s) d x d s+\rho\left(t+t_{0}\right)^{2} .
$$

Thus, for all $(\xi, \eta) \in R^{2}$, from (35), (38) and (39) it follows that

$$
\begin{aligned}
& H(t) \xi^{2}+H^{\prime}(t) \xi \eta+\frac{1}{2 \theta} H^{\prime \prime}(t) \eta^{2} \\
& \geq\left(\int_{0}^{t} \int_{\Omega} a(x, s) u(x, s)^{2} d x d s+\rho\left(t+t_{0}\right)^{2}\right) \xi^{2} \\
& \quad+2 \xi \eta \int_{0}^{t} \int_{\Omega} a(x, s) u(x, s) u_{t}(x, s) d x d s+2 \rho\left(t+t_{0}\right) \xi \eta \\
& \quad+\rho \eta^{2}+\eta^{2} \int_{0}^{t} \int_{\Omega} a(x, s) u_{t}^{2}(x, s) d x d s \geq 0,
\end{aligned}
$$

which implies

$$
\left(H^{\prime}(t)\right)^{2}-\frac{2}{\theta} H(t) H^{\prime \prime}(t) \leq 0
$$

That is,

$$
H(t) H^{\prime \prime}(t)-\frac{\theta}{2}\left(H^{\prime}(t)\right)^{2} \geq 0 .
$$

Then we complete the proof by the standard concavity method (Lemma 2.3) since $\theta>2$.

\section{Competing interests}

The authors declare that they have no competing interests.

\section{Authors' contributions}

The authors declare that the study was realized in collaboration with the same responsibility. All authors read and approved the final manuscript.

\section{Acknowledgements}

We thank the referees for their valuable suggestions which helped us improve the paper so much. This work was supported by the National Natural Science Foundation of China (11171311).

Received: 2 December 2013 Accepted: 13 March 2014 Published: 25 Mar 2014

\section{References}

1. Tan, Z: The reaction-diffusion equation with Lewis function and critical Sobolev exponent. J. Math. Anal. Appl. 2 , 480-495 (2002)

2. Pao, CV: Nonlinear Parabolic and Elliptic Equations. Plenum, New York (1992)

3. Frank-Kamenetskii, DA: Diffusion and Heat Transfer in Chemical Kinetics. Plenum, New York (1969)

4. Gel'fand, IM: Some problems in the theory of quasilinear equations. Usp. Mat. Nauk 2, 87-158 (1959). English transl.: Am. Math. Soc. Transl. (2) 29, 295-381 (1963)

5. Fujuita, $\mathrm{H}$ : On the nonlinear equations $\delta u+e^{u}=0$ and $\partial v / \partial t=\delta v+e^{v}$. Bull. Am. Math. Soc. 75, 132-135 (1969)

6. Peral, I, Vazquez, JL: On the stability or instability of the singular solution of the semilinear heat equation with exponential reaction term. Arch. Ration. Mech. Anal. 129, 201-224 (1995)

7. Pulkkinen, A: Blow-up profiles of solutions for the exponential reaction-diffusion equation. Math. Methods Appl. Sci. 34, 2011-2030 (2011)

8. Tello, J: Stability of steady states of the Cauchy problem for the exponential reaction-diffusion equation. J. Math. Anal. Appl. 324, 381-396 (2006) 
9. Ioku, N: The Cauchy problem for heat equations with exponential nonlinearity. J. Differ. Equ. 251, 1172-1194 (2011)

10. Alves, $\mathrm{CO}$, Cavalcanti, MM: On existence, uniform decay rates and blow up for solutions of the 2-d wave equation with exponential source. Calc. Var. 34, 377-411 (2009)

11. Sattiger, DH: On global solutions of nonlinear hyperbolic equations. Arch. Ration. Mech. Anal. 30, 148-172 (1968)

12. Tsutsumi, M: Existence and nonexistence of global solutions for nonlinear parabolic equations. Publ. Res. Inst. Math. Sci. 8, 211-229 (1972/73)

13. lekhata, R, Suzuki, T: Stable and unstable sets for evolution equations of parabolic and hyperbolic type. Hiroshima Math. J. 26, 475-491 (1996)

14. Levine, HA, Park, SR, Serrin, J: Global existence and nonexistence theorems for quasilinear evolution equations of formally parabolic type. J. Differ. Equ. 142, 212-229 (1998)

15. Messaoudi, SA: A decay result for a quasilinear parabolic system type. In: Elliptic and Parabolic Problems. Progress in Nonlinear Differential Equations and Their Applications, vol. 63, pp. 43-50 (2005)

16. Chen, H, Liu, GW: Global existence and nonexistence theorems for semilinear parabolic equations with conical degeneration. J. Pseud.-Differ. Oper. Appl. 3(3), 329-349 (2012)

17. Moser, J: A sharp form of an inequality by N. Trudinger. Indiana Univ. Math. J. 20, 1077-1092 (1971)

18. Trudinger, NS: On imbeddings into Orlicz spaces and some applications. J. Math. Mech. 17, 473-483 (1967)

19. Nakao, M, Ono, K: Global existence to the Cauchy problem of the semi-linear evolution equations with a nonlinear dissipation. Funkc. Ekvacioj 38, 417-431 (1995)

20. Levine, HA: Some nonexistence and instability theorems for solutions of formally parabolic equations of the form $p u_{t}=-a u+f(u)$. Arch. Ration. Mech. Anal. 51, 371-386 (1973)

10.1186/1687-2770-2014-70

Cite this article as: Dai and Zhang: Energy decay and nonexistence of solution for a reaction-diffusion equation with exponential nonlinearity. Boundary Value Problems 2014, 2014:70

\section{Submit your manuscript to a SpringerOpen ${ }^{\circ}$ journal and benefit from:}

- Convenient online submission

- Rigorous peer review

- Immediate publication on acceptance

- Open access: articles freely available online

- High visibility within the field

- Retaining the copyright to your article 